\title{
A critical evaluation of two-pyroxene thermometry in Adirondack granulites
}

\author{
STEVEN R. BOHLEN \& ERIC J. ESSENE
}

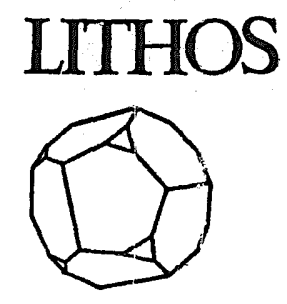

\begin{abstract}
Bohlen, S. R. \& Essene, E. J. 1979: A critical evaluation of two-pyroxene thermoinetry in Adirondack granulites. Lithos 12, 335-345. Oslo. ISSN 0024-4937.

Temperatives have been calculated from the compositions of six:y-five coexisting ortho- and clinopyroxene pairs from the Adirondacks, New York, using calibrations proposed by Wood \& Banno (1973), Ross \& Huebner (1975), Saxena (1976) and Wells (1977), in order to test the accuracy and precision of pyroxene thermometers in metamorphic granulir.s. Calculated temperatures are highly scattered $\left(<600-900^{\circ} \mathrm{C}\right)$ and shov no systematic variation within the Adirondack terrane. Several pyroxene pairs from very localized areas near Colton (N.W. Adirondacks) and Tupper Lake (C. Adirondacks) yield similar scattered temperatures $\left(600-900^{\circ} \mathrm{C}\right)$, which disagree with feldspar and oxide thermometry and are inconsistent with the stability of various silicate and/or sulfide assemblages. All pyroxene thermometer models are sensitive to ferrous-ferric ratios with variations of up to $50^{\circ} \mathrm{C}$ when $\mathrm{Fe}^{3+}$ is estimated from pyroxene stoichiometry for pyroxene compositions obtained by microprobe analysis. These data suggest that the present pyroxene thermometers are sufficiently inconsistent, inaccurate and imprecise that they should not be used as quantitative thermometers in metamorphic rocks.
\end{abstract}

Steven R. Bohlen \& Eric J. Essene, Department of Geology and Mineraiogy, University of Michigan, Ann Arbor, MI 48109, U.S.A.

Coexisting Ca-poor and Ca-rich pyroxenes have long been recognized as a potential geo. thermometer, and consequently there have been several attempts to calibrate the temperature dependence of the solution of enstatite in diopside. Boyd \& Schairer (1964) and Davis \& Boyd (1966) established experimentally the temperature dependence of enstatite-diopsicle solution, which provided the original calibration of the two-pyroxene thermometer for endmember compositions. More recently many additional experiments have been conducted on the pyroxene solvus. Warner \& Luth (19\%4), Nehru \& Wyllie (1974), Howells \& O'Hara (1975), Lindsley \& Dixon (1976) and Mori \& Green $(1975,1976)$ have reported experiments with some tight reversals on the diopside-enstatite solvus. Although there is still some disagreement as to the correct solvus at different P-T, it is evident that these more recent experiments require adjustment of the Davis-Boyd solvus. Other workers have investigated different portions of the pyroxene quadrilateral. Akella \& Boyd (1973) and Lindsiey et al. (1974a, b) performed solvus experiments within the quadrilateral, while Lindsley \& Munoz (1969) and Smith (1972) have reported on phase relations on the join ferrosilite-hedenbergite and within the iron-rich portion of the quadrilateral.
Still other workers, anong them Boyd (1970), Boyd \& Nixon (1973), Akella (1974), Herzberg \& Chapman (1976) and Mori (1977), have examined the effect of $\mathrm{Al}_{2} \mathrm{O}_{3}$ on the solvus. Finally, Ross \& Huebner (1975), Akella (1976), Mysen (1976) and Mori $(1977,1978)$ have attempted to determine the pyroxene solvus with experiments on natural pyroxenes only approximately represented by the quadrilateral. Despite this wealth of experimental data there are no reversed experimental data presently available on the two-pyroxene solvus at rnetamorphic temperatures. Consequently calihracion uf a two-pyroxene solvus for metamorphic rocks typically requires extrapolations is some $200^{\circ} \mathrm{C}$ from higher temperature experimental zeversals. This extrapolation generates significant errors due to relatively wide reversal brackets at higher $\mathrm{T}$ and because of substantial cation ordering effects occurring in pyroxenes at metamorphic T (Saxena \& Ghose 1971), which should widen the solvus relative to disordered pyroxenes. Furthernore, these experimental data indicate that the solvus is ste $c_{2}$ and insensitive to $T$ in the metamorphic temperature range (typically $650-850^{\circ} \mathrm{C}$ for grant lites), implying that very accurate analysis of coexisting pyroxenes is essential for accurate ' $T$ estimates. Despite these potential difficultie; (which also apply to a lesser degree at higher I) 
there have been attempts to calibrate empirically the Ca-rich, Ca-poor pyroxene solvus for all compositions in the quadrilateral. Wood \& Banno (1973) evaluated the then available ex.periments on the pyroxene quadrilateral and obtained an expression of $T$ in terms of

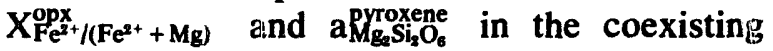
Ca-poor and Ca-rich pyroxenes. They assumed an ideal two-site mixing model allowing for the blocking effect of ordered cations and assumed further that $\mathrm{Mg}$ and $\mathrm{Fe}^{2+}$ are distributed equally on $\mathbf{M}_{2}$ and $\mathbf{M}_{1}$. The Wood-Banno calibration has been applied in several areas (Hewins 1975; Wood 1975; Jayawardena \& Carswell 1976); Wells 1976; Krogh 1977; Ormaasen 1977) with varying degrees of success. Hewins (1975) surveyed two-pyroxene data from Broken Hill and several other granulite terranes and concluded that Wood-Banno pyroxene thermometry was not only precise but accurate to $\pm 30^{\circ} \mathrm{C}$. This is a surprising conclusion in view of the approximations and extrapolations required for the empirical formulation of the thermometer.

In consideration of the more recent experiments on pyroxenes, Wells (1977) reformulated a two-pyroxene thermometer to fit the new experimental data, adapting the form of the WoodBanno equation. Wells noted that the WoodBanno temperatures were seriously in error for magnesian pyroxenes when compared to the later experimental data. Wells (19'7) applied his thermometer to some metamorphic pyroxenes from the literature and concluded that the calculated T's were approximately correct to within $70^{\circ} \mathrm{C}$. Recently Henry \& Medaris (1976 and in Stormer \& Whitney 1977) have modeled the Nehru-Wyllie (1974) and Lindsley-Dixon (1976) solvus data separately in a Wood-Banno type formulation.

Ross \& Huebner (1975) and Saxena (1976) have taken alternate, decidedly different approaches in deriving other two-pyroxene thermometers. Ross \& Huebner (1975) derived a quadrilateral solvus for pyroxenes based on composition-temperature relations of naturally occurring pyroxenes determined in part by homogenization experiments of pyroxenes containing exsolution lamellae. The homogenization experiments are unreversed and hence the precise location of the pigeonite-augite solvus may be somewhat in error. Isotherms at metamorphic temperatures $\left(\leqslant 750^{\circ} \mathrm{C}\right)$ were empirically derived using natural augite compositions from granulite facies rocks. Saxena, on the other hand, has theoretically modeled the activities of pyroxene components using mixing psrameters inferred by Saxena \& Ghose (1971). Saxena's equations for temperature calculations, based in part on compositional data from Ross \& Huebner (1975) and modeling of acitivities, require successive approximations of $\mathrm{T}$ to be obtained with the aid of a computer program. Saxena's temperatures calculated for Bushveld pyroxenes appear to be low as he concedes. The thermometer has not been tested or widely applied in metamorphic rocks, perhaps due to the complexity of the temperature calculations.

Despite the approximations and assumptions; implicit in the formulation of these pyroxene thermometers they have been applied without much critical evaluation of their internal consistency, precision or accuracy. In his calculations on published analyses of two-pyroxene granulites, Hewins (1975) neglected data from the Colton area. Adirondacks (Engel et al. 1964), near the orthopyroxene isograd (for mafic rocks), which yield Wood-Banno temperatures of $850^{\circ} \mathrm{C}$, surely at least $100-150^{\circ} \mathrm{C}$ too high for almost any $\mathrm{T}$ estimate of the amphibolite-granulite boundary (i.e. Turner 1968; Miyashiro 1973; Winkler 1974; Vernon 1976). For the same Adirondack data the pyroxene thermometry of Wells (1977), which presumably should be more arcurate, yields even higher temperatures of $890-920^{\circ} \mathrm{C}$. Lal et al. (1978) compared WoodBanno, Wells and the two Henry-Medaris thermometers in sapphirine granulites and found variations of up to $150^{\circ} \mathrm{C}$ for the sanie pyroxene pair. Stormer \& Whitney (1977) compared several two-pyroxene thermometers with temperature diata obtained from feldspars and Fe-Ti oxides in Brazilian granulites and found substantial disagreements of up to $200^{\circ} \mathrm{C}$. In light of these data there is a strong suggestion that at least some of the two-pyroxene thermometers yield highly suspect results in granulites. Consequently a careful evaluation of these thermometers is necessary.

The Adirondacks are perhaps an ideal field laboratory in which to evaluate pyroxene thermometry critically. Temperature data have been obtained throughout the Adirondacks using elidspar and Fe-Ti oxide thermometry (Stormer 1975; Buddington \& Lindsley 1964; see Bohlen \& Essene 1977, and Fig. 1) and both thermometers are in excellent agreement. These temperature data are further substantiated and con- 
Fig. 1. Outline map of the Precambrian terrane of upper New York State showing Wood-Banno coexisting pyroxene temperatures (using estimates of $\mathrm{Fe}^{3+}$ as calculated from stoichiometry). 0,0 , indicate data obtained by Hoffman (1979), McLelland \& Whitney (1977) and Bohlen, this study, respectively. Isotherms are inferred from oxide and feldspar temperature data, after Bohlen \& Essene 1977; Bohlen et al. 1979.

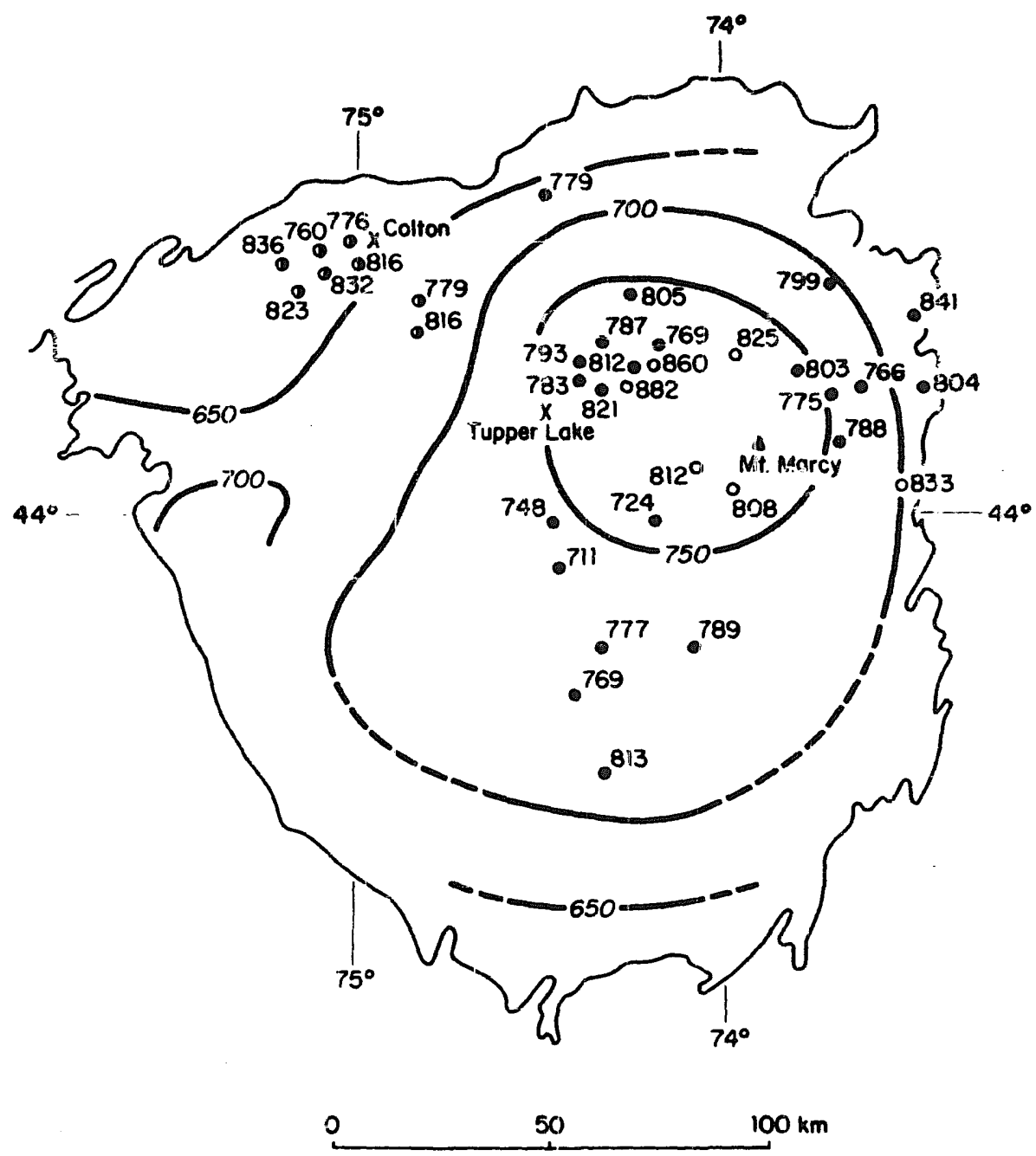

strained by numerous silicate or silicate-carbonate assemblages found in marbles, para- and orthogneisses througiout the Adirondacks (Valley 1977; Essene et al. 1977, 1978; Hoffman 1979; Essene \& Valicy 1979; Valley \& Bohlen 1979) yielding a consistent temperature pattern. Not only are temperatures well known but twopyroxene assemblages are extremely numerous to the east and southeast of the orthopyroxene isograd, located in the northwest Adirondacks (Engel \& Engel 1962; Hoffman 1979). In addition there are now many analyses of Adirondack pyroxenes published by different analysts and using different analytical techniques (Engel et al. 1964; Jaffe et al. 1975; Stoddard 1976; McLelland \& Whitney 1977; Hoffman 1979; Bohlen, this report) with a wide variation in $\mathrm{Mg} /$ $\left(\mathrm{Mg}+\mathrm{Fe}^{2+}\right)$, allowing tests of the compositional effect on the thermometer.

\section{A.nalytical procedure}

Quantitative electrol, microprobe analyses of coexisting orthoand clinopiyroxene were obtained using an ARL-EMX electron microprobe analyzer with wavelength dispersive $\mathrm{LiF}, \mathrm{PE} 1$ and TAP crystal spectrometers. Spectrometer data were corres $e d$ for atomic number, fluorescence, absorption, machine drift and continuous background effects using the Fortan progrim EMPADIR VII (Rucklidge \& Gasparrini 1969). For details of standard operating conditions, standards used in analysis $\neq \neq 1$ additional analytical procedures the reader is referred to Bohlen \& Essene (1976). Pyroxene analyses reported herein were taken from touching grains in the sections which showed textural equilibrium among all pirases.

Pyroxene analyses were normalized about four cations and ferric iron was calcılated from stoichiometry: $\mathrm{Fe}^{3+}=$ $\mathrm{Al}^{\mathrm{V}}-\mathrm{Al}^{\mathrm{VI}}+\mathrm{Na}+\mathrm{K}-27 \mathrm{i}$. Pyroxene data taken from other studies was also nornalized in the same manner so that apparent temperature viriations due to different normalization procedures were itot introduced. In cases where the amount of Al was insuficient to fill the tetrahecial sites we assumed that the deficiency was a result of errors in analysis of $\mathrm{Si}$, ignored it and calculated ferric iron as above (note $O p x$ IN-2, for example). This results $i_{n}$ slightly higher than average $\mathrm{Fe}^{3+}$ estimates, but inspection of Table 1 will show that pyroxenes 
Table 1. Analyses of ortho- and clinopyroxene pairs from the Adirondack Highlands. Temperatures calculated from Wood-Banno assuming $\mathrm{Fe}^{3+}$ calculated from stoichiometry (see text).

\begin{tabular}{|c|c|c|c|c|c|c|c|c|c|c|c|c|}
\hline & $\begin{array}{l}\text { Opx } \\
\text { AS-3 }\end{array}$ & $\begin{array}{l}\text { C'px } \\
\text { AS-3 }\end{array}$ & $\begin{array}{l}\text { Opx } \\
\text { BM-2 }\end{array}$ & $\begin{array}{l}\text { Cpx } \\
\text { BM-2 }\end{array}$ & $\begin{array}{l}\text { Opx } \\
\text { BM-13 }\end{array}$ & $\begin{array}{l}\text { Cpx } \\
\text { BM-13 }\end{array}$ & $\begin{array}{l}\text { Opx } \\
\text { ET-1 }^{\prime}\end{array}$ & $\begin{array}{l}\text { Cpx } \\
\text { ET-1 }\end{array}$ & $\begin{array}{l}\text { Opx } \\
\text { ET-10 }\end{array}$ & $\begin{array}{l}\text { Cpx } \\
\text { ET-10 }\end{array}$ & $\begin{array}{l}\text { Opx } \\
\text { ET-15 }\end{array}$ & $\begin{array}{l}\text { Cpx } \\
\text { ET-15 }\end{array}$ \\
\hline $\mathrm{SiO}_{2}$ & 50.03 & 50.05 & 48.34 & 49.14 & 47.57 & 48.94 & 49.51 & 49.99 & 50.10 & 50.02 & 49.53 & 49.69 \\
\hline $\mathrm{TiO}_{2}$ & 0.08 & (1.29 & 0.07 & 0.15 & 0.08 & 0.19 & 0.05 & 0.18 & 0.10 & 0.29 & 0.05 & 0.09 \\
\hline $\mathrm{Al}_{2} \mathrm{O}_{3}$ & 1.43 & 2.59 & 0.51 & 1.35 & 0.33 & 0.93 & 0.96 & 2.12 & 2.00 & 3.08 & 0.61 & 0.93 \\
\hline $\mathrm{FeO}$ & 31.00 & $12: .87$ & 41.28 & 19.90 & 45.20 & 23.23 & 32.03 & 14.37 & 29.06 & 12.94 & 37.18 & 17.03 \\
\hline MnO & 0.42 & 0.23 & 0.89 & 0.37 & 0.85 & 0.42 & 0.30 & 0.25 & 0.39 & 0.20 & 0.99 & 0.45 \\
\hline $\mathrm{MgO}$ & 16.07 & 11.18 & 7.62 & 6.52 & 4.73 & 4.20 & 14.76 & 10.23 & 17.01 & 11.56 & 10.00 & $8.7 !$ \\
\hline $\mathrm{CaO}$ & 0.56 & 22.14 & 0.89 & 20.96 & 1.00 & 21.13 & 0.50 & 21.33 & 0.52 & 21.02 & 0.62 & 2125 \\
\hline $\mathrm{Na}_{2} \mathrm{O}$ & 0.01 & 0.16 & 0.03 & 0.47 & 0.00 & 0.39 & 0.01 & 0.64 & 0.03 & 0.22 & 0.01 & 0.35 \\
\hline $\mathrm{K}_{2} \mathrm{O}$ & 0.00 & 0.01 & 0.02 & 0.01 & 0.00 & 0.013 & 0.01 & 0.01 & 0.00 & 0.01 & 0.00 & 0.00 \\
\hline Total & 99.50 & 99.52 & 99.65 & 98.87 & 99.76 & 99.43 & 98.13 & 99.12 & 99.21 & 99.34 & 98.99 & 98.54 \\
\hline $\mathrm{Si}$ & 1.950 & 1.907 & 2.001 & 1.948 & 2.010 & 1.965 & 1.975 & 1.919 & 1.943 & 1.905 & 2.020 & 1.950 \\
\hline Al & 0.050 & 0.093 & 0.000 & 0.052 & 0.000 & 0.035 & 0.029 & 0.081 & 0.057 & 0.095 & 0.000 & 0.043 \\
\hline Al & 0.016 & 0.023 & 0.025 & 0.011 & 0.016 & 0.009 & 0.016 & 0.015 & 0.035 & 0.043 & 0.035 & 0.000 \\
\hline $\mathrm{Ti}$ & 0.002 & 0.008 & 0.002 & 0.005 & 0.003 & 0.006 & 0.001 & 0.005 & 0.003 & 0.008 & 0.003 & 0.003 \\
\hline $\mathrm{Fe}^{3+}$ & 0.035 & 0.066 & 0.000 & 0.067 & 0.000 & 0.044 & 0.012 & 0.103 & 0.018 & 0.052 & 0.000 & 0.054 \\
\hline $\mathrm{Mg}$ & 0.934 & 0.635 & 0.470 & 0.385 & 0.298 & 0.251 & 0.878 & 0.585 & 0.983 & 0.656 & 0.609 & 0.509 \\
\hline $\mathrm{Fe}^{2+}$ & 0.976 & 0.344 & 1.429 & 0.593 & 1.597 & 0.736 & 1.056 & 0.358 & 0.925 & 0.360 & 1.269 & 0.505 \\
\hline Mn & 0.014 & 0.007 & $0.03 !$ & 0.012 & 0.030 & 0.014 & 0.010 & 0.008 & 0.013 & 0.007 & 0.035 & 0.015 \\
\hline $\mathrm{Ca}$ & 0.023 & 0.904 & 0.039 & 0.390 & 0.045 & 0.909 & 0.021 & 0.877 & 0.021 & 0.858 & 0.028 & 0.895 \\
\hline $\mathrm{Na}$ & 0.000 & 0.012 & 0.002 & 0.036 & 0.000 & 0.030 & 0.001 & 0.047 & 0.002 & 0.016 & 0.001 & 0.027 \\
\hline $\mathbf{K}$ & 0.000 & 0.000 & 0.001 & 0.000 & 0.000 & 0.000 & 0.001 & 0.000 & 0.000 & 0.000 & 0.000 & 0.000 \\
\hline Wo & 1.2 & 48.0 & 2.0 & 47.6 & 2.3 & 47.9 & 1.1 & 48.2 & 1.1 & 45.8 & 1.5 & 46.9 \\
\hline En & 48.3 & 33.7 & 24.3 & 20.6 & 15.4 & 13.3 & 45.7 & 32.1 & 50.9 & 35.0 & 31.9 & 26.7 \\
\hline Fs & 50.5 & 18.3 & 73.7 & 31.8 & 82.3 & 38.8 & 53.2 & 19.7 & 48.0 & 19.2 & 66.6 & 26.4 \\
\hline $\mathrm{T}^{\circ} \mathrm{C}$ & \multicolumn{2}{|c|}{799} & \multicolumn{2}{|c|}{748} & \multicolumn{2}{|c|}{711} & \multicolumn{2}{|c|}{775} & \multicolumn{2}{|c|}{850} & \multicolumn{2}{|c|}{766} \\
\hline
\end{tabular}

Table / (continued).

\begin{tabular}{|c|c|c|c|c|c|c|c|c|c|c|c|c|}
\hline & $\begin{array}{l}\text { Opx } \\
\text { ET-24 }\end{array}$ & $\begin{array}{l}\text { Cpx } \\
\text { ET-24 }\end{array}$ & $\begin{array}{l}\text { Opx } \\
\text { IN-2 }\end{array}$ & $\begin{array}{l}\text { Cpx } \\
\text { IN-2 }\end{array}$ & $\begin{array}{l}\text { Opx } \\
\text { IN-8 }\end{array}$ & $\begin{array}{l}\text { Cpx } \\
\text { IN-8 }\end{array}$ & $\begin{array}{l}\text { Opx } \\
\text { IN-11 }\end{array}$ & $\begin{array}{l}\text { Cpx } \\
\text { IN-11 }\end{array}$ & $\begin{array}{l}\text { Opx } \\
\text { LL-8 }\end{array}$ & $\begin{array}{l}\text { Cpx } \\
\text { LL-8 }\end{array}$ & $\begin{array}{l}\text { Opx } \\
\text { MM-18 }\end{array}$ & $\begin{array}{l}\text { Cpx } \\
\text { MM-18 }\end{array}$ \\
\hline $\mathrm{SiO}_{2}$ & 50.20 & 51.25 & 49.77 & 50.11 & 47.62 & 49.70 & 46.80 & 49.92 & 52.17 & 50.85 & 48.59 & 49.82 \\
\hline $\mathrm{TiO}_{2}$ & 0.17 & 0.29 & 0.06 & 0.54 & 0.09 & 0.18 & 0.11 & 0.20 & 0.04 & 0.41 & 0.09 & 0.24 \\
\hline $\mathrm{Al}_{2} \mathrm{O}_{3}$ & 0.90 & 2.37 & 1.02 & 4.67 & 0.55 & 1.55 & 0.39 & 1.15 & 1.28 & 3.14 & 0.48 & 1.61 \\
\hline $\mathrm{FeO}$ & 31.94 & 13.57 & 29.83 & 11.56 & 39.49 & 19.13 & 43.56 & 21.85 & 26.68 & 10.38 & 38.46 & 18.54 \\
\hline $\mathrm{MnO}$ & 0.32 & 0.15 & 1.05 & 0.40 & 1.37 & 0.63 & 1.57 & 0.72 & 0.64 & 0.26 & 0.47 & 0.29 \\
\hline $\mathrm{MgO}$ & 15.46 & 10.21 & 17.71 & 10.77 & 8.89 & 7.33 & 6.13 & 5.27 & 18.66 & 12.12 & 10.21 & 8.21 \\
\hline $\mathrm{CaO}$ & 0.61 & 21.71 & 0.40 & 20.70 & 0.75 & 29.33 & 0.82 & 20.81 & 0.44 & 21.74 & 0.84 & $20.1 f$ \\
\hline $\mathrm{Na}_{2} \mathrm{O}$ & 0.04 & 0.62 & 0.01 & 0.78 & 0.05 & 0.64 & 0.00 & 0.57 & 0.01 & 0.48 & 0.02 & $0.4 ;$ \\
\hline $\mathrm{K}_{2} \mathrm{O}$ & 0.01 & 0.00 & 0.00 & 0.00 & 0.00 & 0.00 & 0.00 & 0.00 & 0.00 & 0.00 & 0.01 & 0.00 \\
\hline Tciai & 99.65 & 100.17 & 99.85 & 99.53 & 98.81 & 99.49 & 99.38 & 100.49 & 99.92 & 99.38 & 99.17 & 99.35 \\
\hline $\mathrm{Si}$ & 1.966 & 1.945 & 1.919 & 1.894 & 1.970 & 1.946 & 1.968 & 1.966 & 1.990 & 1.918 & 1.984 & $1 . \mathcal{S}_{46}$ \\
\hline Al & 0.034 & 0.055 & 0.046 & 0.106 & 0.027 & 0.054 & 0.019 & 0.034 & 0.010 & 0.082 & 0.016 & 0.154 \\
\hline $\mathrm{Al}$ & 0.007 & 0.051 & 0.000 & 0.102 & 0.000 & 0.018 & 0.000 & 0.018 & 0.047 & 0.057 & 0.007 & 0.120 \\
\hline $\mathrm{Ti}$ & 0.005 & 0.008 & 0.002 & 0.015 & 0.003 & 0.005 & 0.003 & 0.006 & 0.001 & 0.012 & 0.003 & 0.107 \\
\hline $\mathrm{Fe}^{3+}$ & 0.019 & 0.033 & 0.050 & 0.031 & 0.025 & 0.074 & 0.012 & 0.048 & 0.000 & 0.036 & (0.005 & 0.056 \\
\hline $\mathbf{M g}$ & 0.903 & 0.577 & 1.017 & 0.607 & 0.548 & 0.428 & 0.384 & 0.310 & 1.061 & 0.682 & 0.622 & 0.478 \\
\hline $\mathrm{Fe}^{2+}$ & 1.027 & 0.398 & 0.911 & 0.335 & 1.342 & 0.553 & 1.520 & 0.672 & 0.851 & 0.291 & 1.308 & 0.549 \\
\hline $\mathbf{M n}$ & $0.01 !$ & 0.005 & 0.033 & 0.013 & 0.048 & 0.021 & 0.056 & 0.025 & 0.021 & 0.008 & 0.017 & 0.009 \\
\hline $\mathrm{Ca}$ & 0.026 & 0.883 & 0.016 & 0.839 & 0.033 & 0.853 & 0.037 & 0.878 & 0.918 & 0.878 & 0.037 & 0.844 \\
\hline $\mathrm{Na}$ & 0.002 & 0.045 & 0.008 & 0.057 & 0.00 & 0.048 & 0.000 & 0.044 & 0.103 & 0.035 & $0.00 ?$ & 0.036 \\
\hline $\mathbf{K}$ & 0.000 & 0.000 & 0.000 & 0.000 & 0.0010 & 0.000 & 0.000 & 0.000 & 0.000 & 0.000 & 0.600 & 0.000 \\
\hline$w_{0}$ & 1.3 & 47.5 & 0.8 & 47.1 & 1.7 & 46.5 & 1.9 & 47.2 & 0.5 & 47.4 & 1.9 & 45.1 \\
\hline En & 46.2 & 31.1 & 52.3 & 34.1 & 28.5 & 23.3 & 19.8 & 16.7 & 55.0 & 36.8 & 31.6 & 25.5 \\
\hline Fs & 52.5 & 21.4 & 46.9 & 18.8 & 69.8 & 30.2 & 78.3 & 36.1 & 44.1 & 15.8 & 66.5 & 29.4 \\
\hline $\mathrm{T}^{\circ} \mathrm{C}$ & \multicolumn{2}{|c|}{788} & \multicolumn{2}{|c|}{813} & \multicolumn{2}{|c|}{769} & \multicolumn{2}{|c|}{777} & \multicolumn{2}{|c|}{821} & \multicolumn{2}{|c|}{803} \\
\hline
\end{tabular}


Table I (continued).

\begin{tabular}{|c|c|c|c|c|c|c|c|c|c|c|c|c|}
\hline & $\begin{array}{l}\text { Opx } \\
N-3\end{array}$ & $\begin{array}{l}\text { Cpx } \\
\mathbb{N}-3\end{array}$ & $\begin{array}{l}\text { Of } 1 x \\
\text { PII-4 }\end{array}$ & $\begin{array}{l}\text { Cpx } \\
\text { PH-4 }\end{array}$ & $\begin{array}{l}\text { Opx } \\
\text { SC-8 }\end{array}$ & $\begin{array}{l}\text { Cpx } \\
\text { SC-8 }\end{array}$ & $\begin{array}{l}\text { Opx } \\
\text { SL-5 }\end{array}$ & $\begin{array}{l}\text { Cpx } \\
\text { SL-5 }\end{array}$ & $\begin{array}{l}\text { Opx } \\
\text { SL-13 }\end{array}$ & $\begin{array}{l}\text { Cpx } \\
\text { SL-13 }\end{array}$ & $\begin{array}{l}\text { Opx } \\
\text { SL-26 }\end{array}$ & $\begin{array}{l}\text { Cpx } \\
\text { SL.26 }\end{array}$ \\
\hline $\begin{array}{l}\mathrm{SiO}_{2} \\
\mathrm{TiO}_{2} \\
\mathrm{Al}_{2} \mathrm{O}_{3} \\
\mathrm{FeO} \\
\mathrm{MnO} \\
\mathrm{MgO} \\
\mathrm{CaO} \\
\mathrm{Na}_{2} \mathrm{O} \\
\mathrm{K}_{2} \mathrm{O} \\
\text { Total }\end{array}$ & $\begin{array}{r}50.33 \\
0.06 \\
1.51 \\
29.44 \\
0.33 \\
16.96 \\
0.53 \\
0.01 \\
0.00 \\
99.17\end{array}$ & $\begin{array}{r}50.95 \\
0.18 \\
2.45 \\
10.72 \\
0.13 \\
12.10 \\
22.78 \\
0.67 \\
0.01 \\
99.99\end{array}$ & $\begin{array}{r}50.44 \\
0.06 \\
1.66 \\
30.22 \\
0.31 \\
16.87 \\
0.50 \\
0.00 \\
0.00 \\
100.06\end{array}$ & $\begin{array}{r}50.50 \\
0.81 \\
3.00 \\
11.05 \\
0.17 \\
11.54 \\
22.27 \\
0.20 \\
0.00 \\
99.54\end{array}$ & $\begin{array}{r}50.08 \\
0.08 \\
1.44 \\
29.64 \\
1.06 \\
15.94 \\
0.57 \\
0.01 \\
0.00 \\
98.82\end{array}$ & $\begin{array}{r}49.96 \\
0.23 \\
2.49 \\
13.12 \\
0.47 \\
10.96 \\
21.67 \\
0.53 \\
0.00 \\
99.43\end{array}$ & $\begin{array}{r}50.28 \\
0.05 \\
1.71 \\
31.24 \\
0.45 \\
15.56 \\
0.57 \\
0.01 \\
0.00 \\
99.87\end{array}$ & $\begin{array}{r}49.34 \\
0.50 \\
3.04 \\
13.96 \\
0.30 \\
10.38 \\
21.15 \\
0.19 \\
0.00 \\
98.86\end{array}$ & $\begin{array}{r}50.11 \\
0.08 \\
1.29 \\
27.62 \\
0.53 \\
18.43 \\
0.43 \\
0.02 \\
0.00 \\
98.51\end{array}$ & $\begin{array}{r}51.25 \\
0.27 \\
2.34 \\
10.97 \\
0.13 \\
12.53 \\
22.33 \\
0.51 \\
0.00 \\
100.33\end{array}$ & $\begin{array}{r}45.75 \\
0.09 \\
0.32 \\
49.36 \\
0.82 \\
1.83 \\
0.93 \\
0.04 \\
0.00 \\
99.14\end{array}$ & $\begin{array}{r}49.55 \\
0.28 \\
0.90 \\
27.99 \\
0.47 \\
1.55 \\
19.03 \\
0.57 \\
0.00 \\
100.34\end{array}$ \\
\hline $\begin{array}{l}\text { Si } \\
\mathbf{A l}\end{array}$ & $\begin{array}{l}1.956 \\
0.044\end{array}$ & $\begin{array}{l}1.910 \\
0.090\end{array}$ & $\begin{array}{l}1.946 \\
0.054\end{array}$ & $\begin{array}{l}1.915 \\
0.085\end{array}$ & $\begin{array}{l}1.965 \\
0.035\end{array}$ & $\begin{array}{l}1.903 \\
0.097\end{array}$ & $\begin{array}{l}1.959 \\
0.04 !\end{array}$ & $\begin{array}{l}1.902 \\
0.098\end{array}$ & $\begin{array}{l}1.942 \\
0058\end{array}$ & $\begin{array}{l}1.914 \\
0.086\end{array}$ & $\begin{array}{l}1.989 \\
0.011\end{array}$ & $\begin{array}{l}2.014 \\
0.000\end{array}$ \\
\hline $\begin{array}{l}\mathrm{Al} \\
\mathrm{Ti} \\
\mathrm{Fe}^{3+} \\
\mathbf{M} \mathbf{B} \\
\mathrm{Fe}^{2+} \\
\mathrm{Mn}^{2+} \\
\mathrm{Ca} \\
\mathrm{Na} \\
\mathbf{K}\end{array}$ & $\begin{array}{l}0.025 \\
0.002 \\
0.015 \\
0.983 \\
0.942 \\
0.011 \\
0.022 \\
0.000 \\
0.000\end{array}$ & $\begin{array}{l}0.018 \\
0.005 \\
0.110 \\
0.676 \\
0.226 \\
0.004 \\
0.915 \\
0.048 \\
0.000\end{array}$ & $\begin{array}{l}0.022 \\
0.002 \\
0.028 \\
0.970 \\
0.947 \\
0.010 \\
0.021 \\
0.000 \\
0.000\end{array}$ & $\begin{array}{l}0.049 \\
0.023 \\
0.004 \\
0.652 \\
0.347 \\
0.005 \\
0.905 \\
0.014 \\
0.000\end{array}$ & $\begin{array}{l}0.032 \\
0.002 \\
0.000 \\
0.933 \\
0.973 \\
0.035 \\
0.024 \\
0.001 \\
0.000\end{array}$ & $\begin{array}{l}0.015 \\
0.006 \\
0.109 \\
0.622 \\
0.309 \\
0.015 \\
0.884 \\
0.039 \\
0.000\end{array}$ & $\begin{array}{l}0.027 \\
0.002 \\
0.000 \\
0.904 \\
1.018 \\
0.015 \\
0.024 \\
0.000 \\
0.000\end{array}$ & $\begin{array}{l}\mathbf{c . 0 4 0} \\
\mathbf{0 . 0 1 5} \\
0.042 \\
0.597 \\
0.408 \\
0.010 \\
0.374 \\
0.014 \\
0.000\end{array}$ & $\begin{array}{l}0.001 \\
0.602 \\
0.055 \\
1.065 \\
0.838 \\
0.017 \\
0.018 \\
0.002 \\
0.000\end{array}$ & $\begin{array}{l}0.017 \\
0.008 \\
0.090 \\
0.697 \\
0.253 \\
0.004 \\
0.894 \\
0.037 \\
0.000\end{array}$ & $\begin{array}{l}0.005 \\
0.003 \\
0.004 \\
0.119 \\
1.79 \\
0.030 \\
0.043 \\
0.004 \\
0.000\end{array}$ & $\begin{array}{l}0.043 \\
0.009 \\
0.000 \\
0.094 \\
0.951 \\
0.016 \\
0.829 \\
0.045 \\
0.000\end{array}$ \\
\hline $\begin{array}{l}\text { Wo } \\
\text { En } \\
\text { Fs }\end{array}$ & $\begin{array}{r}1.1 \\
50.5 \\
48.4\end{array}$ & $\begin{array}{l}50.4 \\
37.2 \\
12.4\end{array}$ & $\begin{array}{r}1.1 \\
50.0 \\
48.9\end{array}$ & $\begin{array}{l}47.5 \\
34.2 \\
18.3\end{array}$ & $\begin{array}{r}1.2 \\
48.3 \\
50.5\end{array}$ & $\begin{array}{l}48.7 \\
34.3 \\
17.0\end{array}$ & $\begin{array}{r}1.2 \\
46.5 \\
52.3\end{array}$ & $\begin{array}{l}46.5 \\
31.8 \\
21.7\end{array}$ & $\begin{array}{r}0.9 \\
55.5 \\
43.6\end{array}$ & $\begin{array}{l}48.5 \\
37.8 \\
13.7\end{array}$ & $\begin{array}{r}2.2 \\
6.1 \\
91.7\end{array}$ & $\begin{array}{r}44.2 \\
5.1 \\
50.7\end{array}$ \\
\hline $\mathbf{T}^{\circ} \mathrm{C}$ & \multicolumn{2}{|c|}{724} & \multicolumn{2}{|c|}{804} & \multicolumn{2}{|c|}{779} & \multicolumn{2}{|c|}{812} & \multicolumn{2}{|c|}{805} & \multicolumn{2}{|c|}{769} \\
\hline
\end{tabular}

Table I (continued).

\begin{tabular}{|c|c|c|c|c|c|c|c|c|c|c|}
\hline & $\begin{array}{l}\text { Opx } \\
\text { SR-18 }\end{array}$ & $\begin{array}{l}\text { Cpx } \\
\text { SR-18 }\end{array}$ & $\begin{array}{l}\text { Opx } \\
\text { SR-29 }\end{array}$ & $\begin{array}{l}\text { Cpx } \\
\text { SR-29 }\end{array}$ & $\begin{array}{l}\text { Opx } \\
\text { SR-31 }\end{array}$ & $\begin{array}{l}\text { Cpx } \\
\text { SR-31 }\end{array}$ & $\begin{array}{l}\text { Opx } \\
\text { TL-4 }\end{array}$ & $\begin{array}{l}\text { Cpx } \\
\text { TI_-4 }\end{array}$ & $\begin{array}{l}\text { Opx } \\
\text { W-9 }\end{array}$ & $\begin{array}{l}\text { Cpx } \\
\text { W-9 }\end{array}$ \\
\hline $\begin{array}{l}\mathrm{SiO}_{2} \\
\mathrm{TiO}_{2} \\
\mathrm{Al}_{2} \mathrm{O}_{3} \\
\mathrm{FeO} \\
\mathrm{MnO} \\
\mathrm{MgO} \\
\mathrm{CaO} \\
\mathrm{Na}_{2} \mathrm{O} \\
\mathrm{K}_{2} \mathrm{O} \\
\text { Total }\end{array}$ & $\begin{array}{r}50.52 \\
0.04 \\
i .56 \\
30.92 \\
0.65 \\
15.54 \\
0.55 \\
0.00 \\
0.00 \\
99.78\end{array}$ & $\begin{array}{r}49.76 \\
0.34 \\
2.33 \\
13.36 \\
0.35 \\
10.68 \\
21.98 \\
0.19 \\
0.00 \\
98.99\end{array}$ & $\begin{array}{r}44.96 \\
0.06 \\
0.38 \\
48.73 \\
0.99 \\
2.95 \\
0.68 \\
0.01 \\
0.00 \\
98.76\end{array}$ & $\begin{array}{r}48.52 \\
0.19 \\
0.97 \\
23.31 \\
0.48 \\
5.10 \\
19.88 \\
0.61 \\
0.00 \\
99.06\end{array}$ & $\begin{array}{r}46.94 \\
0.10 \\
0.36 \\
43.67 \\
0.48 \\
7.16 \\
0.83 \\
0.00 \\
0.00 \\
99.54\end{array}$ & $\begin{array}{r}51.00 \\
0.26 \\
1.29 \\
23.28 \\
0.27 \\
5.14 \\
19.02 \\
0.51 \\
0.00 \\
100.77\end{array}$ & $\begin{array}{r}46.66 \\
0.06 \\
0.46 \\
42.91 \\
1.16 \\
7.17 \\
0.78 \\
0.00 \\
0.00 \\
99.20\end{array}$ & $\begin{array}{r}49.03 \\
0.19 \\
1.02 \\
23.15 \\
0.70 \\
5.98 \\
19.32 \\
0.61 \\
0.00 \\
100.00\end{array}$ & $\begin{array}{r}50.30 \\
0.05 \\
1.84 \\
28.39 \\
0.24 \\
17.78 \\
0.52 \\
0.01 \\
0.00 \\
99.13\end{array}$ & $\begin{array}{r}49.48 \\
0.46 \\
3.68 \\
12.69 \\
0.19 \\
11.04 \\
20.92 \\
0.38 \\
0.01 \\
98.85\end{array}$ \\
\hline $\begin{array}{l}\text { Si } \\
\text { Al }\end{array}$ & $\begin{array}{l}1.971 \\
0.029\end{array}$ & $\begin{array}{l}1.913 \\
0.087\end{array}$ & $\begin{array}{l}1.951 \\
0.019\end{array}$ & $\begin{array}{l}1.946 \\
0.046\end{array}$ & $\begin{array}{l}1.957 \\
0.018\end{array}$ & $\begin{array}{l}2.011 \\
0.000\end{array}$ & $\begin{array}{l}1.951 \\
0.023\end{array}$ & $\begin{array}{l}1.939 \\
0.048\end{array}$ & $\begin{array}{l}1.944 \\
0.056\end{array}$ & $\begin{array}{l}1.893 \\
0.107\end{array}$ \\
\hline $\begin{array}{l}\mathbf{A l} \\
\mathrm{Ti} \\
\mathbf{F e}^{3+} \\
\mathbf{M g} \\
\mathrm{Fe}^{2+} \\
\mathrm{Mn} \\
\mathrm{Ca} \\
\mathrm{Na} \\
\mathrm{F}\end{array}$ & $\begin{array}{l}0.043 \\
0.001 \\
0.000 \\
0.903 \\
1.009 \\
0.021 \\
0.023 \\
0.000 \\
0.000\end{array}$ & $\begin{array}{l}0.019 \\
0.010 \\
0.062 \\
0.612 \\
0.367 \\
0.011 \\
0.906 \\
0.014 \\
0.000\end{array}$ & $\begin{array}{l}0.000 \\
0.002 \\
0.016 \\
0.191 \\
1.752 \\
0.036 \\
0.032 \\
0.001 \\
0.000\end{array}$ & $\begin{array}{l}0.000 \\
0.006 \\
0.081 \\
0.184 \\
0.820 \\
0.016 \\
0.854 \\
0.047 \\
0.000\end{array}$ & $\begin{array}{l}0.600 \\
0.603 \\
0.612 \\
0.445 \\
1.512 \\
0.617 \\
0.037 \\
0.000 \\
0.000\end{array}$ & $\begin{array}{l}0.050 \\
0.008 \\
0.000 \\
0.302 \\
0.768 \\
0.009 \\
0.804 \\
0.039 \\
0.000\end{array}$ & $\begin{array}{l}0.000 \\
0.002 \\
0.019 \\
0.447 \\
1.482 \\
0.041 \\
0.035 \\
0.000 \\
0.000\end{array}$ & $\begin{array}{l}0.000 \\
0.006 \\
0.082 \\
0.353 \\
0.684 \\
0.024 \\
0.819 \\
0.046 \\
0.000\end{array}$ & $\begin{array}{l}0.028 \\
0.001 \\
0.026 \\
1.024 \\
0.891 \\
0.008 \\
0.022 \\
0.000 \\
0.00\end{array}$ & $\begin{array}{l}0.059 \\
0.013 \\
0.050 \\
0.630 \\
0.356 \\
0.006 \\
0.858 \\
0.028 \\
0.300\end{array}$ \\
\hline $\begin{array}{l}\text { Wo } \\
\text { En } \\
\text { Fs }\end{array}$ & $\begin{array}{r}1.2 \\
46.7 \\
52.1\end{array}$ & $\begin{array}{l}48.1 \\
32.5 \\
19.4\end{array}$ & $\begin{array}{r}1.6 \\
9.7 \\
88.7\end{array}$ & $\begin{array}{r}46.0 \\
9.9 \\
44.1\end{array}$ & $\begin{array}{r}1.9 \\
22.3 \\
75.8\end{array}$ & $\begin{array}{l}42.9 \\
16.1 \\
41.0\end{array}$ & $\begin{array}{r}1.8 \\
22.7 \\
75.5\end{array}$ & $\begin{array}{l}44.1 \\
19.0 \\
36.9\end{array}$ & $\begin{array}{r}1.1 \\
52.9 \\
46.0\end{array}$ & $\begin{array}{l}45.5 \\
34.2 \\
19.3\end{array}$ \\
\hline $\mathrm{T}^{\circ} \mathrm{C}$ & \multicolumn{2}{|c|}{787} & \multicolumn{2}{|c|}{793} & \multicolumn{2}{|c|}{783} & \multicolumn{2}{|c|}{789} & \multicolumn{2}{|c|}{841} \\
\hline
\end{tabular}


Table 2. Comparison of temperature data obtained using several pyroxene thermometers and feldspar and Fe-Ti oxide thermometers from assemblages found near Colton (Adironilack Lowlands), Tupper Lake and Mount Marcy (both Adirondack Highlands). Pyroxene data for Mount Marcy taken from Jaffe et al. (1975), Colton data from Stoddard (1976) and Hoffman (1979). Feldspar and Fe-Ti oxide data from Bohlen \& Essene (1977) and unp sblished data.

\begin{tabular}{|c|c|c|c|c|}
\hline Modei & & Colton & Tupper $L k$. & Mt. Marcy \\
\hline \multirow{2}{*}{ Wood-Eianno } & $\{$ Total Fe & $746-827$ & $764-880$ & $722-786$ \\
\hline & $\left\{\right.$ Est $\mathrm{Fe}^{3+}$ & $759-836$ & $769-882$ & $746-840$ \\
\hline \multirow{2}{*}{ Wells } & $\int$ Total Fe & $755-87$ & $758-883$ & $713-817$ \\
\hline & I Est $\mathrm{Fe}^{3+}$ & $763-879$ & $764-900$ & $746-872$ \\
\hline \multirow{4}{*}{$\begin{array}{l}\text { Henry-Medaris } \\
\text { (Nehru--Wyllie) } \\
\text { Henry-Medaris } \\
\text { (Lindsley-Dixon) }\end{array}$} & $\{$ Total Fe & $604-735$ & $664-757$ & $550-773$ \\
\hline & Est $\mathrm{Fe}^{3+}$ & $612-742$ & $679-776$ & $622-797$ \\
\hline & $\{$ Total Fe & $611-793$ & $694-842$ & $629-880$ \\
\hline & $\left\{\right.$ Est $\mathrm{Fe}^{3+}$ & $622-806$ & $716-872$ & $638-918$ \\
\hline Ross-Huebrier & $\left\{\begin{array}{l}\text { Total } \mathbf{F e} \\
\text { Est } \mathrm{Fe}^{3+}\end{array}\right.$ & $\begin{array}{l}\quad 600-650 \\
<600\end{array}$ & $\begin{array}{l}<600-750 \\
<600-680\end{array}$ & $\begin{array}{r}600-800 \\
<600-700\end{array}$ \\
\hline \multicolumn{2}{|l|}{ Feldspars } & $630-660$ & $710-750$ & 750 \\
\hline \multicolumn{2}{|l|}{ Fe-Ti osides } & $\begin{array}{l}\text { insufficient } \\
\text { data }\end{array}$ & $720-79)$ & 780 \\
\hline
\end{tabular}

with apparent $\mathrm{Si}$ errors are not substantially different from average calculated $\mathrm{Fe}^{3+}$. Ferric iron estimates reported compare favorably with direct determinations for pyroxenes reported by Engel et al. (1964). However a $1 \%$ error in analysis of $\mathrm{SiO}_{2}$ gives a typical error in calculated $\mathrm{Fe}^{3+}$ of roughly $10-40 \%$. This in turn yields $\mathrm{T}$ variations of less than $40^{\circ} \mathrm{C}$ for most coexisting pyroxene thermometers rejorted below. Because of the problem of estimation of ferric iron from microprobe analyses we have calculated pyroxene temperatures (1) assurning total $\mathrm{Fe}=\mathrm{Fe}^{2+}$ and (2) with $\mathrm{Fe}^{3+}$ calculated as above. The results and conclusions which follow are unaffected by the uncertainty in estimates of $\mathrm{Fe}^{3+}$.

\section{Analytical results and discussion}

Analytical data for coexisting pyroxenes are compiled in Table 1. The temperatures given in Table 1 have been callculated using Wood-Banno and have been plotted on an outline map of the Adirondacis in Fig. 1. Inspection of Fig. 1 shows immediately that the Wood-Banno temperatures are highly variable even among pyroxene pairs obtained from closely spaced outcrops. The temperature values are generally quite high, typically $50-150^{\circ} \mathrm{C}$ above both oxide and feldspar temperature data. In addition comparison of Wood-Banino pyroxene temperatures across the Adirondacks suggests that metamorphic grade is not changing significantly from the northwest Lowlands to the central Highlands. However, these conclusions are contrary to the observations of other workers Engel \& Engel 1962; Buddington 1963, 1966; De'Waard 1966, 1967; Bohlen \& Essene 1977; Bohlen ef al. 1979; Hoffman 1979), which indicate that metamorphic temperatures increase from the Lowlands to the Highlands but remain rather constant over broad areas of the Highlands (note the $700^{\circ} \mathrm{C}$ isotherm in Fig. 1). In addition, high pyroxene temperature values are wholly inconsistent with numerous phlogopite-calcitequartz, tremolite-calcite-quartz and grossularrich garnet-quartz assemblages found in marbles throughout the Adirondacks (Valley \& Essene 1976, 1977) and also inconsistent with muscovite-quartz if the small modal amounts of muscovite $(<5 \%)$ typically found in Adirondack Lowlands (northwest Adirondacks) para- and orthogneisses are primary as has been inferred by previous workers (Engel \& Engel 1962; De Waard 1967). Thus it seems that the WoodBanno thermometer is both imprecise and inaccurate in Adirondack granulites. Calculation of temperatures taking $\Sigma \mathrm{Fe}=\mathrm{Fe}^{2+}$ (ignoring $\mathrm{Fe}^{3+}$ estimations) yields somewhat lower temperatures (typically $10-30^{\circ} \mathrm{C}$ ) but does not alter the conclusions concerning the lack of precision and accuracy. The results of other pyroxene thermometers (Wells, Henry-Medaris and RossHuebner) yield similarly scattered and apparently inaccurate results. Table 2 shows a comparison of the results obtained from several pyroxene thermometers with $\mathrm{Fe}-\mathrm{Ti}$ oxide and two-feluspar data for three restricted localities in the Adirondacks. Analyses of twenty-one, nine and eight pyroxene pairs were obtained from areas no greater than $100 \mathrm{sq} . \mathrm{km}$ near Colton, Tupper Lake and Mount Marcy, respectively. Within these localized areas metamorphic tern- 
Fig. 2. Comparison of coexisting pyroxene temperatures calculated using Wood-Banno $(a, c)$ and Wells $(b, d)$ assuming $\Sigma \mathrm{Fe}=\mathrm{Fe}^{2+}$ in the northwest Adirondacks (near Colton - a, b) and in the central Adirondacks (pairs from within the $700^{\circ} \mathrm{C}$ is(3therm, c, d). In $2 a$ and $2 \mathrm{~b}$ and $\square$ indicate $\mathrm{T}$ calculated from data by Hoffman (1979) and Stoddard (1976) respectively. In $2 \mathrm{c}$ and $2 \mathrm{~d}$ $\odot, \square$, indicate $T$ calculated from data of Jaffe et al. (1975), McLelland \& Whitney (1971) and Bohlen, this study, respectively.
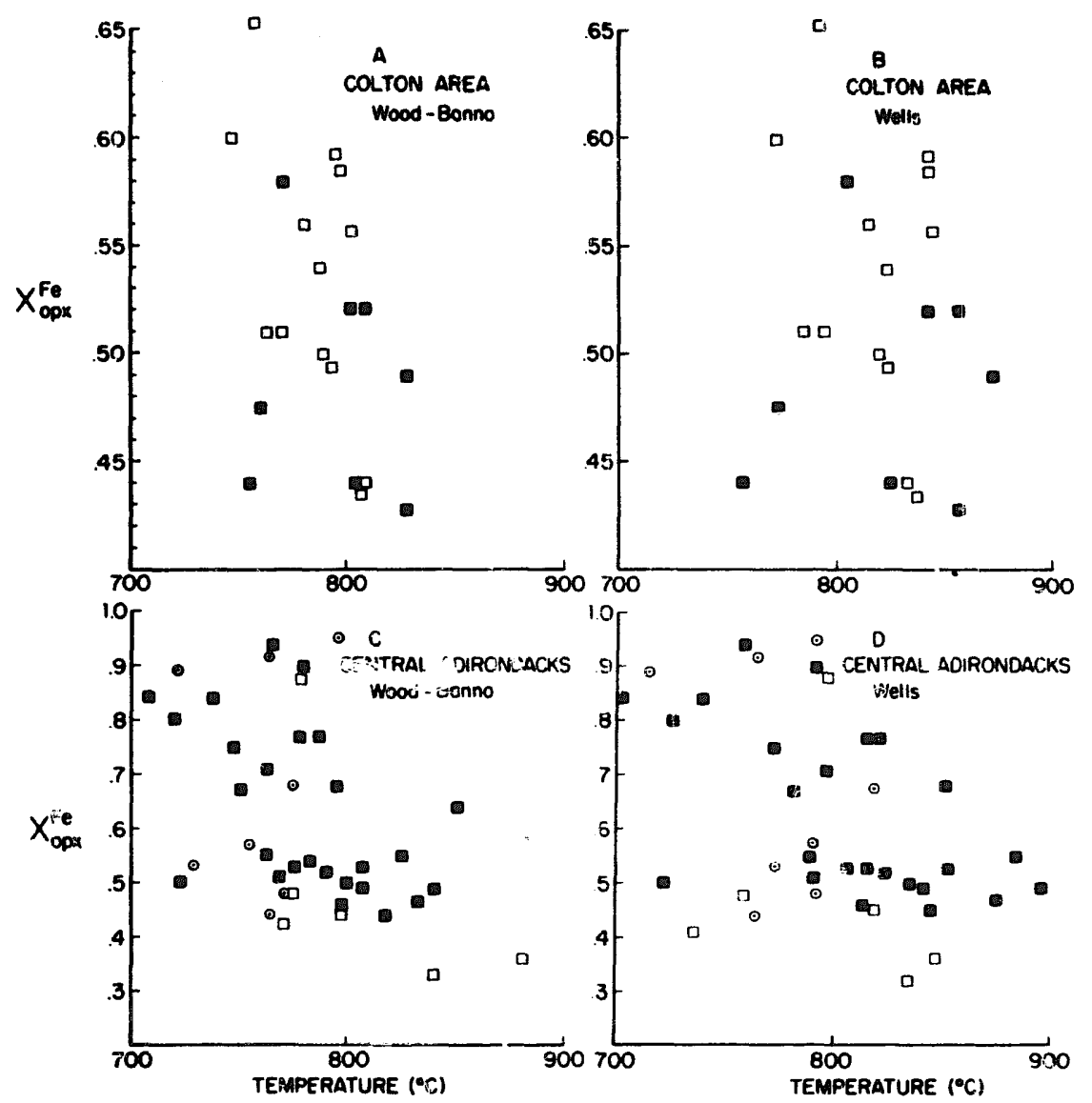

peratures are presumably not varying significantly. Table 2 shows that the five pyroxene thermometers cisagree among themselves by as much as $350^{\circ} \mathrm{C}$ and are different from the feldspar and oxide temperatures by up to $200^{\circ} \mathrm{C}$. Table 2 also shows the dramatic effect estimation of $\mathrm{Fe}^{3+}$ content has on the calculated temperatures. Henry \& Medaris (written communication, 1978) no longer consider the calibration of their pyroxene thermometers (Henry \& Medaris 1976) to be correct and decided against publishing the thermometers in their present form. We shall not consider the results from the Henry-Medaris thermometers, and their use by other works is to be avoided even though the equations have been published in Stormer \& Whitney (197'7). The data for Wood-Banno (1973) and Wells (1977) thermometry of Colton area pyroxenes are shown in Figs. $2 a$ and $2 b$, respectively. Pyroxenes were cobtained from amphibolites and other ortho- and paragneisses. Note that it is not just one aberrant pyroxene pair which gives rise to the wide temperature variation listed in Table 2. Temperatures calcu- lated for Colton pyroxenes average $788^{\circ}$ (WoodBanno, $\Sigma \mathrm{Fe}=\mathrm{Fe}^{2+}$ ), $799^{\circ}$ (Wood-Banno with estimated $\mathrm{Fe}^{3+}$ ), $819^{\circ}$ (Wells, $\Sigma \mathrm{Fe}=\mathrm{Fe}^{2+}$ ) and $832^{\circ}$ (Wells with estimated $\mathrm{Fe}^{3+}$ ), $150-200^{\circ} \mathrm{C}$ above feldspar temperatures for the same area. Any of these pyroxene temperatures are also inconsistent with assemblages of phlogopitecalcite-quartz and tremolite-calcite-quartz found nearby (Valley \& Essene 1976, 1977; Valley 1977) and biotite (annite 40-60) stabilisy for $\mathrm{P}_{\mathrm{H} 2 \mathrm{O}}<\mathrm{P}_{\text {soild }}$ conditions (Essene et al. 1978). Ross-Huebner thermometry yields 'temperature values which agree reasonably with feldspar and oxide data for $\Sigma \mathrm{Fe}=\mathrm{Fe}^{2+}$, however, when $\mathrm{Fe}^{3+}$ is estimated the inferred temperatures are reduced to values below $600^{\circ} \mathrm{C}$, which appear to be too low. Temperatures obtained from calculations using the data of Engel et al. (1964) for pyroxenes near Colton yield distinctly higher temperatures (840-860' Wood-Banno; 890-920 Wells; 730-750 Ross-Huebner) than those calculated from the data of Stoddard (1976) and Hoffman (1979). The compositions of pyroxenes analyzed by Stoddard (1976) and Hoffman (1979) 
Table 3. Analyses of a typical ortho- and clinopyroxene pair from the Colton area containing very thin, minor exsolution lamellae.

\begin{tabular}{|c|c|c|c|c|}
\hline & $\begin{array}{l}\text { Cpx } \\
\text { GVR-53 }\end{array}$ & $\begin{array}{l}\text { Cpx } \\
\text { GVR-53 }\end{array}$ & $\begin{array}{l}\text { Opx } \\
\text { GVR-53 } \\
\quad-\text { Reir }\end{array}$ & $\begin{array}{c}\text { Cpx } \\
\text { GVR-53 } \\
\text { ategrated }\end{array}$ \\
\hline $\mathrm{SiO}_{2}$ & 50.56 & 51.12 & 50.26 & 51.31 \\
\hline $\mathrm{TiO}_{2}$ & 0.05 & 0.17 & 0.07 & 0.15 \\
\hline $\mathrm{Al}_{2} \mathrm{O}_{3}$ & 1.29 & 2.20 & 1.38 & 1.98 \\
\hline $\mathrm{FeO}$ & 31.33 & 13.58 & 31.41 & 13.78 \\
\hline MnO & 0.85 & 0.36 & 0.65 & 0.41 \\
\hline $\mathrm{MgO}$ & 16.31 & 11.40 & 16.07 & 11.66 \\
\hline $\mathrm{CaO}$ & 0.56 & 21.48 & 0.76 & 21.31 \\
\hline $\mathrm{Na}_{2} \mathrm{O}$ & 0.04 & 0.50 & 0.03 & 0.42 \\
\hline $\mathrm{K}_{2} \mathrm{O}$ & 0.00 & 0.00 & 0.00 & 0.00 \\
\hline Total & 100.99 & 100.81 & 100.63 & 101.02 \\
\hline $\mathrm{Si}$ & 1.943 & 1.920 & 1.941 & 1.924 \\
\hline Al & 0.057 & 0.080 & 0.059 & 0.076 \\
\hline Al & 0.002 & 0.017 & 0.004 & 0.012 \\
\hline $\mathrm{Ti}$ & $(1.001$ & 0.005 & 0.002 & 0.004 \\
\hline $\mathrm{Fe}^{3+}$ & 0.056 & 0.089 & $0.05 \hat{3}$ & 0.087 \\
\hline $\mathbf{M g}$ & 0.935 & 0.638 & 0.925 & 0.652 \\
\hline $\mathrm{Fe}^{2+}$ & 0.952 & 0.338 & 0.961 & 0.345 \\
\hline Mn & 0.028 & 0.012 & 0.021 & 0.013 \\
\hline $\mathrm{Ca}$ & 0.023 & 0.865 & 0.032 & 0.856 \\
\hline $\mathrm{Na}$ & 0.003 & 0.036 & 0.002 & 0.031 \\
\hline $\mathbf{K}$ & 0.000 & 0.000 & 0.000 & 0.000 \\
\hline Wo & 1.2 & 47.0 & 1.7 & 46.2 \\
\hline En & 49.0 & 34.7 & 48.2 & 35.2 \\
\hline Fs & 49.8 & 18.3 & 50.1 & 18.6 \\
\hline
\end{tabular}

by electron microprobe analysis differ markedly in wollastonite content from the analyses of Engel et al. (1964), which were obtained using bulk separates. In particular the Engel et al. (1964) orthopyroxene data have 2-3 mole\% greater and the clinopyroxenes $2-4$ mole\% less wollastonite component than similar pyroxenes analyzed by iricroprobe. These pyroxenes frequently show very small homogeneously distributed exsolution lamellae. Even though Engel et al. (1964) claim that they saw no exsolution lanellae in the pyroxenes they analyzed, we were concerned that analyses obtained from bulk separates yield metamorphically equilibrated compositions (including the lamellae) and the microprobe data yield postmetamorphic compositions (host compositions excluding lamellae). We have tested this by reintegrating pyroxenes with obvious, thin exsolution lamellae (using the wide-beam microprobe technique described in Bohlen \& Essene 1978) and comparing their compositions with analyses of pyroxenes in the same rock which show no exsolution iamellae. Wollastonite content of pyroxene compositions obtained using these two techniques differ by less than 0.5 mole\% (see Table 3), suggesting that the microprobe analyses are giving relatively precise and accurate mineral compositions despite the minor, thin exsolution lamellae. In view of these data it seems likely that the data of Engel et al. (1964) are slightly in error probably due to small amounts $(<3 \%$ ) of contamination of orthopyroxene in the clinopyroxene separates and clinopyroxene in orthopyroxene splits. This would easily explain the differences in the pyroxene analyses and hence account for the dissonant temperature values calculated from compositions obtained by microprobe and bulk separate analyses.

Comparison of pyroxene thermometry data with $\mathrm{T}$ inferred from feldspars, oxides and silicate assemblages for Tupper Lake and Mount Marcy yield discrepancies similar to those observed for Colton data. Again the temperature values inferred from any individual two-pyroxene thermometer vary by as much as $200^{\circ} \mathrm{C}$ and except for a few temperatures the pyroxene thermometry does not agree well with other data. In the Mount Marcy area the assemblage monticellite-wollastonite-akermanite (Essene \& Valley 1979; Valley \& Essenc, in preparation) fixes $\mathrm{T}=780 \pm 30^{\circ} \mathrm{C}$ in excellent agreement with feldspar and oxide data, yet pyroxene temperature data give widely varying and generally inaccurate results.

In addition to problems of precision and accuracy the empirically derived Wood-Banno anc Wells thermometers seem also to have a com. positional dependence on calculated temperatures. If the feldspar and oxide ternperature data are correct then most pyroxenes within the $700^{\circ} \mathrm{C}$ isotherm (Fig. 1) in the central Adirondack Highlands have equilibrated at 741$) \pm 40^{\circ} \mathrm{C}$. If we make the simplifying assumption that they are in fact isothermal and then plot $T$ vs. $X_{\mathrm{opx}}^{\mathrm{Fe}}$ (see Figs. 2c and 2d) a substantial compositional effect on $T$ is seen, despite rather wide scatter in the data. Consideration of estirnated $\mathrm{Fe}^{3+}$ reduces neither the scatter nor the apparent $T$ dependence on composition. The Ross-Huebner thermometer does not appear to show a strong T-composition dependence (Fig. 3). However, the Ross--Huebner thermometer is quite sensitive to $\mathrm{Fe}^{3+}$ estimates and seems in either case to yield generally low temperatures. In consideration of these problems it appears that the WoodBanno, Wells and Ross-Huebner calibrations 
Fig. 3. Coexisting pyroxene temperatures obtained for central Adirondacks pyroxenes (pairs within the $700^{\circ} \mathrm{C}$ isotherm) using RossHuebner (1975). (a) T obtained assuming $\Sigma \mathrm{Fe}=\mathrm{Fe}^{2+}$. (b) $\mathrm{T}$ obtained assuming estimated $\mathrm{Fe}^{3+}$.
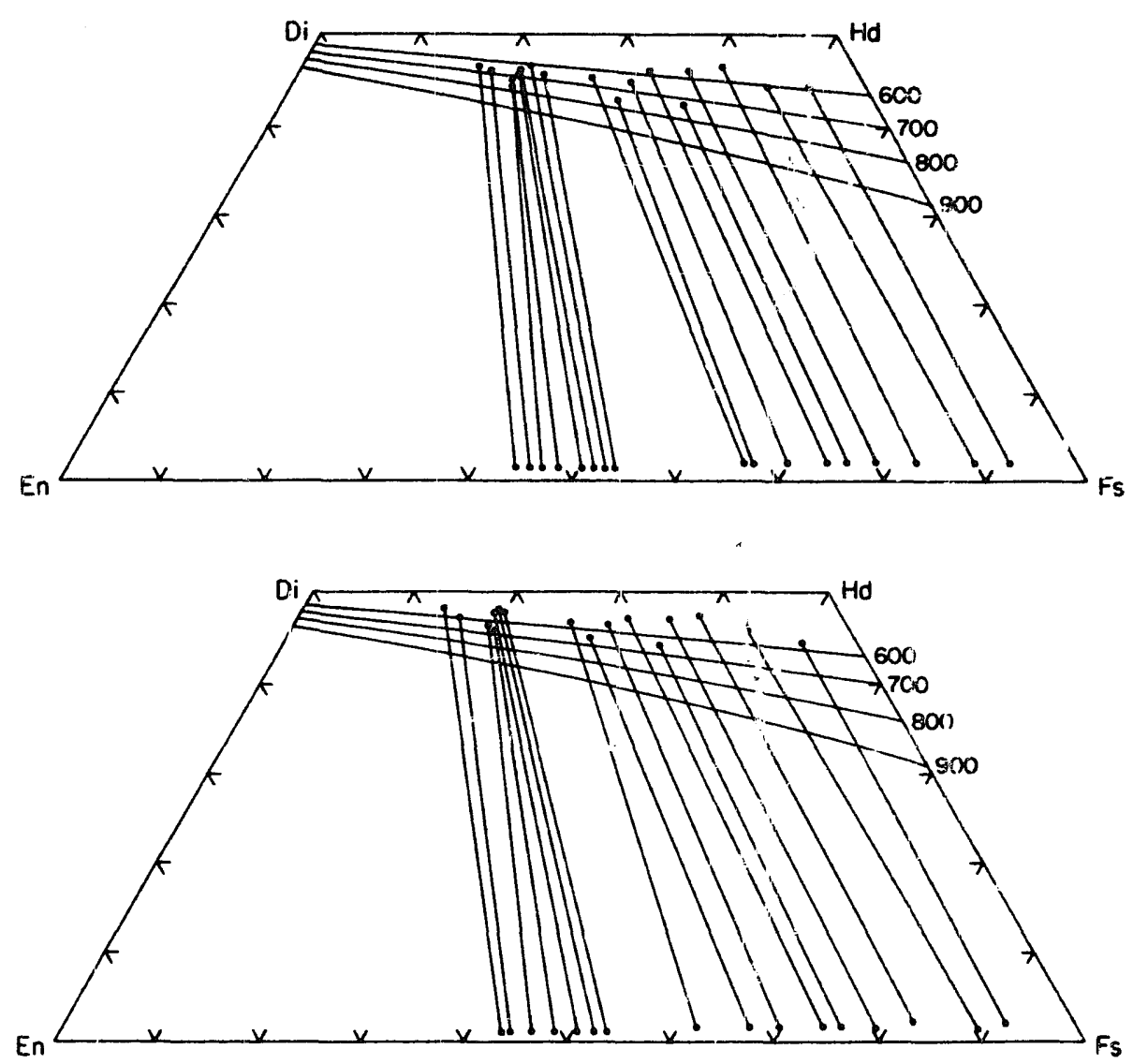

are not useful as quantitative thermometers in metamorphic rocks.

We have also attempted to apply the Saxena (1976) and the Saxena \& Nehru (1975) thermometers to Adirondack pyroxenes. Unfortunately the Saxena (1976) model gives no solution for many pyroxene pairs because calculations often yield the natural log of a negative number. When solutions were obtained the resultant temperature values were highly scattered $\left(700-900^{\circ} \mathrm{C}\right.$ ) and extremely sensitive to $\mathrm{Fe}^{3+}$ estimates with temperature shifts of up to $150^{\circ} \mathrm{C}$ for $\Sigma \mathrm{Fe}=\mathrm{Fe}^{2+}$ vs. estimated $\mathrm{Fe}^{3+}$. For relatively Mg-rich pyroxenes the Saxena \& Nehru (1975) pyroxenc thermometer (1975) also gave scattered results but generally high temperatures $\left(815-975^{\circ} \mathrm{C}\right)$. Saxena \& IVehru state that their thermometer is only approximate and should only be applied to magnesian pyroxenes. In short both the Saxena-Nehru (1975) and Saxena (1976) thermometers appear also to be inadequate for quantitative use in metamorphic terranes.

There are, no doubt, many reasons for tine apparent inaccuracy and imprecision in these two-pyroxene thermometers. Surely a large portion of the uncertainty stems from a lack of experimental reversals on end-member compositions and compositions within the quadrilateral at metamorphic temperatures. The lack of tight reversals at higher $\mathrm{T}$ prevents accurate extrapo. lation to lower $\mathbf{T}$. Other problems also piague these thermometers. Recently Evans \& Trommsdorff (1978) and Henry \& Medaris (written communication, 1979) have shown that a plot of $\ln \mathrm{K}$ vs. $1 / \mathrm{T}$ for exchange of $\mathrm{Mg}_{2} \mathrm{Si}_{2} \mathrm{O}_{6}$ in experimental solvus data is a curve rather than linear as had been previously assumed by Wood \& Banno (1973) and Wells (1977). Assuming a straight-line fit results in substantial overestimation of $T$ for pyroxenes equilibrated at metamorphic T. However, due, again, to the lack of tight reversals the location of this calibration curve is quite uncertain at low temperatures. In addition, simplifying assumptions concerning the site occupancy of $\mathrm{Mg}-\mathrm{Fe}$ and the effect of additional components must anso adversely affect the results. Another scurce of error which may be underestimated is analytical error. In addition to errors in estimated $\mathrm{Fe}^{3+}$ 
which were discussed previously, analytical errors, especially in $\mathrm{Ca}, \mathrm{Mg}$ and $\mathrm{Fe}$ will significantly affect the results of the two-pyroxene thermometers because of the greatly reduced $T$ dependence of the solvus width at low temperatures.

In short, it appears that the lack of precision and accuracy of tw/o-pyroxene thermometry in metamorphic rocks is due to several experimental, analytical and theoretical uncertainties. The results of this study indicate that two pyroxene thermometry in metamorphic rocks will at best yield only very approximate temperature data which should not and cannot be used as the only thermometer in a given metamorphic terrane.

Acknowiedgements. - The writers are grateful for and acknowledge the support of National Science Foundation Grant Nos. EAR 75-22388 and EAR 70-23568 to EJE, Geological Society of America Penrose Grant No. 2003-75 to SRB and the Turner Fund, the University of Michigan. The electron microprobe facilities at the University of Michigan are supervised by Professor Wilbur C. Bigelow of the Department of Metallurgy and Material Science whose cooperation made this study possible. Several members of his staff, particularly L. F. Allard, P. H. Hollingsworth and A. J. Mardinly gave valuable assistance during data collection. The writers thank Johy ritzgerald of the Department of Earth Sciences, Monash University, Australia, for his help in writing the computer programs used in calculating Saxena temperatures. We especially want to thank Dr. Gordon Medaris, Jr., of the Department of Geology and Geophysics, the University of Wisconsin-Madison. for his review of the manuscript. Mr. Derwin Bell drafted the figures. Contribution No. 351 from the Mineralogical Laboratory Department of Geology and Mineralogy. University of Michigan, Ann Arbor, MI 48109, U.S.A

\section{References}

Akella, J. 1974: Solubility of $\mathrm{Al}_{2} \mathrm{O}_{3}$ in orthopyroxene coexisting with garnet and clinopyroxene for compositions on the diopside-pyrope join in the system $\mathrm{CaSiO}_{3}-\mathrm{MgSiO}_{3}-\mathrm{Al}_{2} \mathrm{O}_{3}$. Carnegie Inst. Wash. Year Book 73, 273-278.

Akella, J. 1976: Garnet pyroxene equilibria in the system $\mathrm{CaSin}_{n}-\mathrm{MgSiO}_{3}-\mathrm{Al}_{2} \mathrm{O}_{3}$ and in a natural mixture. Am. Mircral 6I, 585.598.

Akelia, J. \& Boyd, F. R. 1973: Effect of pressure on the composition of coexisting pyroxtres and garnet in the system $\mathrm{CaSiO}_{3}-\mathrm{MgSiO}_{3}-\mathrm{FeSiO}_{3}-\mathrm{Ca}^{\prime} \mathrm{TiAl}_{2} \mathrm{O}_{6}$. Carnegie Inst. Wash. Year Book 72, 523-526.

Bohlen, S. R. \& Essene, E. J. 1977: Feldspar and oxide thermometry of granulites in the Adirondack Highlands. Conirib. Mineral. Petrol. 62, 152-169.

Bohle⿰, S. R. \& Essene, E. J. 1978: Igneous pyroxenes from metamorphosed anorthosite massifs. Contrib. Mineral. Petrol. 65. 433-442.

Bohler., S. R., Essene, E. ¿. \& Hoffman, K. S. 1979: Feldspar and oxide thermometry in the Adirondacks: an update. Bull. Gieol. Soc. Am., in press.

Boyd, F. R. 1970. Garnet peridotites in the system $\mathrm{CaSiO}_{3}-$ $\mathrm{MgSiO}_{3}-\mathrm{Al}_{2} \mathrm{O}_{3}$. Mineral. Soc. Am. Spec. Pap. 3, 63-75.
Boyd, F. R. \& Nixon, P. H. 1973: Structure of the upper mantle beneath Lesotho. Carnegie Inst. Wash. Year Book $72,431-445$.

Boyd, F. R. \& Schairer, J. F. 1964: 'The system $\mathrm{MgSiO}_{3}-$ $\mathrm{CaMlgSi}_{2} \mathrm{O}_{6}$. J. Petrol. 5, 275-309.

Buddington, A. F. 1963: Isograds and the role of $\mathrm{H}_{2} \mathrm{O}$ in metamorphic facies of orthogneisses of the northwest Adirondack area, New York. Bull. Geol. Soc. Am. 74, 1155-1182.

Buddington, A. F. 1966: The occurrence of garnet in the granulite facies terrane of the Adirondack Highlands: A discussion. J. Petrol. 7, 331-335.

Buddington, A. F. \& Lindsley, D. H. 1964: Iron-titanium oxidues and their synthetic equivalents. J. Petrol. 5, 310-357.

Davis, B. T. C. \& Boyd, F. R. 1966: The join $\mathrm{Mg}_{2} \mathrm{Si}_{2} \mathrm{O}_{6}-$ $\mathrm{CaMgSi}_{2} \mathrm{O}_{6}$ at 30 kilobars pressure and its application to pyroxenes from kimberlites. J. Geophys. Res. 7I, 35673576.

DeWaard, D. 1966: The biotite-cordierite-almandine subfacies of the hornblende-granulite facies. Can. Mineral. 8, 481492.

DeWaard, D. 1967: Absolute P-T conditions of granulitefacies metmorphism in the Adirondacks. Kon. Ned. Akad. Weter. - Amsterdam Ser. B, 70, 400-410.

Engel, A. E. J. \& Engel, C. G. 1962: Progressive metamorphism of amphibolites northwest Adirondack Mountains, New York. Geol. Soc. Am. Buddington Volume, 37-82.

Engel, A. E. J., Engel, C. G. \& Havens, R. G. 1964: Mineralogy of amphibolite interlayers in the gneiss complex, northwest Adirondack Mountains, New York. J. Geol. 72, 13]-156.

Essene, E. J., Bohlen, S. R. \& Valley, J. W. 1977: Regional metamorphism in the Adirondacks. Geot. Soc. Am. Abstr. with Program 9, 260-261.

Essene, E. J., Bohlen, S. R. \& Valley, J. W. 1978: Determination of regional water fugacities in the Adirondacks. Geol. Soc. Am. Abstr. with Program 10, 397-398.

Es'sene, E. J. \& Valley, J. IV. 1979: High pressure akermanite in the Adirondacks. Geol. Soc. Am. Abstr. with Program II, 11.

Evans, B. W. \& Trommsdorff, V. 1978: Petrogenesis of garnet lherzolite, Cima Di Gagnone, Lepontine Alps. Earth Planet. Sci. Lett. 40, 333-348.

Henry. D. J. \& Medaris, L. G. 1976: Application of pyroxene and olivine-spinel geothermometers to the alpine peridotites in southwestern Oregon, Geol. Soc. Am. Abstr. with Program 8, 913-914.

Herzberg, C. T. \& Chapman, N. A. 1976: Clinopyroxene geothermometry of spinei-liherzolites. Am. Mineral. 61 , 626-637.

Hewins, R. H. 1975: Pyroxene geothermometry of some granulite facies rocks. Contrib. Mineral. Petrol. 50, 205-209.

Hoffman, K. S. 1979: A reevaiuation of the orthopyroxene isograd in the northwest Adirondacks. MS thesis, the University of Michigan.

Howells, S. \& O'Hara, M. J. 1975: Palaeogeotherms and the diopside-enstatitr sulyıis. Nature 254, 406-408.

Jaffe, Fi. W., Robinson, P., T:acy, R. J. \& Ross, M. 2975: Orientation of pigeonite exsolution lamellae in metamorphic augite: correlation with composition and calculated optimal phase boundaries. Am. MAinerai. Af, 9-28.

Jayawardena, D. E. De S. \& Carsweil, D. A. 1976: The geochemistry of 'charnockites' and their constituent ferromagnesian minerals from the Preciambrian of south-east Sri Lanka (Ceylon). Mineral. Mag. 40, 54!-553.

Krogh, E. J. 1977: Origin and metamorphism of iron forma- 
tions and associated rocks, Lofoten-Vesterålen, $\mathbf{N}$. Norway. I. The Vestpolltind Fe-Mn deposit, Lithos 10, 243-255.

Lal, R. K., Ackermand, D., Seifert, F. \& Halder, S. K. 1978: Chemographic relationships in sapphirine-bearing rocks from Sonapahar, Assam, India. Contrib. Mineral. Petrol. 67, 169-187.

Lindsley, D. H. \& Dixor., S. A. 1976: Diopside-enstatite equilibria at 850 to $1400^{\circ} \mathrm{C}, 5$ to $35 \mathrm{~kb}$. Am. J. Sci. 276, 1285-1301.

Lindsley, D. H., King, H. E., Jr. \& Turnock, A. C. 1974a: Phase relations in the pyroxene quadrilateral at $980^{\circ} \mathrm{C}$ and 15 kbar. Geol. Soc. Am. Abstr. with Program 6, 846-847.

Lindsley, D. H., King, H. E., Jr. \& Turnock, A. C. 1974b: Compositions of synthetic augite and hypersthene coexisting at $810^{\circ} \mathrm{C}$ : application to pyroxenes from lunar highland rocks. Geophys. Res. Lett. I, 134-136.

Lindsley, D. H. \& Munoz, J. L. 1969: Subsolidus relations along the join hedenbergite-ferrosilite. Am. J. Sci. 267-A, 295-324.

McLelland, J. M. \& Whitney, P. R. 1977: The origin of garnet in the anorthosite-charnockite suite of the Adirondacks. Contrib. Mineral. Petrol. 60, 161-181.

Miyashiro, A. 1973: Metamorphism and Metamorphic Belts, George Allen \& Unwin Ltd., London.

Mori, T. 1977. Geothermometry of spinel therzolites. Contrib. Mineral. Petrol. 59, 261-279.

Mori, T. 1978: Experimental study of pyroxene equilibria in the system CaO-MgO-FeO-SiO ${ }_{2}$. J. Geol. 19, 45-65.

Mori, T. \& Green, D. H. 1975: Pyroxenes in the system $\mathrm{Mg}_{2} \mathrm{Si}_{2} \mathrm{O}_{6}-\mathrm{CaMgSi}_{2} \mathrm{O}_{6}$ at high pressure. Earth Planet. Sci. Lett. 26, 277--286.

Mori, T. \& Green, D. H. 1976: Subsolidus equilibria between pyroxenes in the $\mathrm{CaO}-\mathrm{MgO}-\mathrm{SiO}_{2}$ system at high pressures and temperatures. Am. Mineral. 61, 616-625.

Mysen, B. O. 1976: Experimental determination of some geochemical parameters relating to conditions of equilibration of peridotite in the upper mantle. Alm. Mineral. 6I, 677-683.

Nehru, C. E. \& Wyllie, P. J. 1974: Electron microprobe measurements of pyroxenes coexisting with $\mathrm{H}_{2} \mathrm{O}$ undersaturated liquids on the join $\mathrm{CaMgSi}_{2} \mathrm{O}_{6}-\mathrm{Mg}_{2} \mathrm{Si}_{2} \mathrm{O}_{6}-\mathrm{H}_{2} \mathrm{O}$ at 30 kilobars with application to geothermometry. Contrib. Mineral. Petrol. 48, 221-228.

Ormaasen, D. E. 1977: Petrology of the Hopen mangeritecharnockite intrusion, Lototen, north Norway. Lithos 10. 291-310.

Ross, M. \& Huebner, J. S. 1975: A pyroxene geothermometer based on composition-temperature relationships of naturally occurring orthopyroxene, pigeonite and augite. Extended Abstract, Internat. Conf. Geothermometry Geobarometry, Penn. St. Univ.

Rucklidge, J. C. \& Gasparrini, E. L. 1969: Specifications of a complete program for processing electron microprobe data: EMPADR VII. Dept. of Geology, Univ. of Toronto, unpublished circular.
Saxena, S. K. 1976: Two-pyroxene geothermometer: a model with an approxinate sulution. Am. Mineral. 61, 643-652.

Saxena, S. K. \& Ghose, S. 1971: $\mathrm{Mg}^{2+}-\mathrm{Fe}^{2+}$ order-disorder and the thermodynamics of the orthopyroxene crystalline solution. Am. Mineral. 56, 532-559.

Saxena, S. K. \& Nehru, C. E. 1975: Enstatite-diopside solvus and geothermometry. Contrib. Mineral. Petrol. 49, $259-267$.

Smith, D. 1972: Stability of iron-rich pyroxene in the sys $\{\mathrm{em}$ $\mathrm{CaSiO}_{3}-\mathrm{FeSiO}_{3}-\mathrm{MgSiO}_{3}$. Am. Mineral. 57, 1413-1428.

Stoddard, E. F. 1976: Granulite facies metamorphism in the Colton-Rainbow Falls Area, northwest Adirondacks, New York. Ph.D. thesis, University of California, Los Angeles.

Stormer, J. C., Jr. 1975: A practical two-feldspar geothermometer. Am. Mineral. 60, 667-674.

Stormer, J. C., Jr. \& Whitney, J. A. 1977: Two-feldspar geothermometer in granulite facies metamorphic rocks. Contrib. Mineral. Petrol. 65, 123-133.

Turner, F. J. 1968: Metamorphic Petrology: Mineralogical and Field Aspects. McGraw-Hill, New York.

Valley, J. W. 1977: Calc-silicate in Grenville marble, Adirondack Mountains, New York, Ms. thesis, the University of Michigan.

Valley, J. W. \& Bohlen, S. R. 1979: A petrogenetic grid for Adirondack metamorphism. Geol. Soc. Am. Abstr. with Program II, 51.

Valley, J. W. \& Essene, E. J. 1976: Calc-silicate reactions in Grenville marble, New York. Geol. Soc. Am. Abstr. with Program 8, 1151-1152.

Valley, J. W. \& Essene, E. J. 1977: Regional metamorphic wollastonite in the Adirondacks. Geol. Soc. Am. Abstr. with Program 9, 260-261.

Vernon, R. H. 1976: Metamorphic Processes, George Allen \& Unwin Ltd., London.

Warner, R. D. \& Luth, W. C. 1974: The diopside orthoenstatite two-phase region in the system $\mathrm{C}_{1} \mathrm{MgSi}_{2} \mathrm{O}_{6}-\mathrm{Mg}_{2} \mathrm{Si}_{2} \mathrm{O}_{6}$. Am. Mineral. 59, 98-109.

Wells, P. R. A. 1976: Late Archean netamorphism in the Buksefjorden region, southwest Greenland. Contrib. Mineral. Petrol. 56, 229-242.

Wells, P. R. A. 1977: Pyroxene thermometry in simple and complex systems. Contrib. Mineral. Petrol. 62, 129-139.

Winkler, H. G. F. 1974: Petrogenesis of Metamorphic Rocks. Springer-Verlag.

Wood, B. J. 1975: The influence of pressure, temperature and bulk composition on the appearance of garnet in orthogneisses - an example from South Harris, Scotland. Earth Planet. Sci. Lett. 26, 299-311.

Wood, B. J. \& Banno, S. 1973: Garnet-orthopyroxene and orthopyroxene-clinopyroxene relationships in simple and complex systems. Contrib. Mineral. Petrol. 42, 109-124.

Accepted for publication May 1979

Printed October 1979 